\title{
Relationship between brachial artery flow-mediated dilatation, hyperemic shear stress, and the metabolic syndrome
}

\author{
Lawrence M Title ${ }^{1}$, Evan Lonn², Francois Charbonneau ${ }^{3}$, Marinda Fung ${ }^{3}$, Kieren J Mather ${ }^{4}$, Subodh Verma ${ }^{5}$ and \\ Todd J Anderson ${ }^{3}$
}

\begin{abstract}
Metabolic syndrome (MetSyn) may predispose to cardiovascular disease (CVD) by causing vascular dysfunction. This study aimed to determine the association of MetSyn with vascular function, as assessed by brachial artery flow-mediated dilatation (FMD) and hyperemic shear stress (HSS). A total of 1,417 male firefighters without established diabetes and CVD were classified for MetSyn, according to the National Cholesterol Education Program Adult Treatment Panel III (NCEP) definition. MetSyn was present in 267 individuals (19\%). Although FMD was lower in those with versus without MetSyn $(8.1 \pm 4.1$ vs $8.7 \pm 4.0 \% ; p=0.02)$, this was not significant after adjusting for baseline differences (age, smoking, and brachial artery diameter) $(p=0.2)$. However, HSS was significantly lower in those with versus without MetSyn $\left(72.0 \pm 27.8\right.$ vs $\left.80.9 \pm 24.8 \mathrm{dyne} / \mathrm{cm}^{2} ; p<0.001\right)$, and there was a significant inverse graded relationship with the number of NCEP criteria present (mean HSS for those with $0,1,2,3,4$, and 5 criteria: $83.2 \pm 22.5,82.2 \pm 24.7,76.5 \pm 27.2,74.3 \pm 27.4,66.5 \pm$ $28.4,67.1 \pm 27.6 \mathrm{dyne} / \mathrm{cm}^{2} ; p<0.001$ for trend). The individual NCEP criteria of abdominal obesity, systolic hypertension, and impaired fasting glucose were independent predictors for HSS. In conclusion, MetSyn was not associated with impaired FMD. Alternatively, HSS, a measure of microvascular function, was significantly lower in those with MetSyn. Thus, MetSyn may contribute to CVD by causing microvascular dysfunction.
\end{abstract}

Key words: endothelium; insulin resistance; metabolic syndrome; obesity; vasodilation

\section{Introduction}

The clustering of central obesity, insulin resistance, dyslipidemia, hypertension, and dysglycemia was first recognized by Reaven in 1988, and was eventually called the metabolic syndrome (MetSyn). ${ }^{1}$ In 2002, the National Cholesterol Education Program Adult Treatment Panel III (NCEP) published clinical criteria for the identification of the MetSyn, ${ }^{2}$ which were subsequently modified in $2004 .{ }^{3}$ Although the utility of the NCEP MetSyn definition in clinical care remains controversial, ${ }^{4,5}$ it has proven useful in epidemiologic studies where the

\footnotetext{
${ }^{1}$ Division of Cardiology at Dalhousie University, Halifax, Nova Scotia, Canada; ${ }^{2}$ Division of Cardiology at McMaster University, Hamilton, Ontario, Canada; ${ }^{3}$ Division of Cardiology at University of Calgary, Calgary, Alberta, Canada; ${ }^{4}$ Division of Endocrinology at Indiana University, Indianapolis, Indiana, USA; ${ }^{5}$ Division of Cardiovascular Surgery, University of Toronto, Toronto, Ontario, Canada

Correspondence to: Lawrence Title, Queen Elizabeth II Health Sciences Centre, Division of Cardiology, 6896-1796 Summer St, Halifax, NS, Canada B3H 3A7. Email: 1title@dal.ca
}

presence of the MetSyn has been associated with an increased risk for the development of cardiovascular disease (CVD) and type 2 diabetes, itself an independent risk factor for CVD. ${ }^{6-8}$ Moreover, a number of studies have shown associations between the presence of MetSyn and subclinical atherosclerosis, as assessed by carotid ultrasound, ${ }^{9}$ cardiac computed tomography, ${ }^{10}$ and coronary angiography, ${ }^{11}$ suggesting MetSyn is a risk factor for atherosclerosis.

The mechanism by which the MetSyn may predispose to atherosclerotic CVD remains speculative. However, it has been proposed that the MetSyn and/or insulin resistance may promote endothelial vascular dysfunction, which plays a key role in atherogenesis. ${ }^{12,13}$ Peripheral macrovascular endothelial function can be non-invasively assessed using high-resolution ultrasound by measuring flowmediated dilatation (FMD) of a conduit vessel, such as the brachial artery, in response to hyperemic shear stress (HSS). FMD is attenuated in individuals at risk of CVD or with established atherosclerosis, and impaired FMD may be predictive of future cardiovascular events. ${ }^{14-16}$ Alternatively, 
microvascular function can be assessed by a number of techniques to measure changes in regional blood flow in response to either pharmacologic or physical stimuli that are partly related to endotheliumdependent vasodilation of resistance vessels. ${ }^{17}$ HSS, which is the stimulus for inducing FMD, can be measured with vascular Doppler during FMD testing. HSS represents a non-invasive measure of microvascular function, which has been shown to correlate with coronary risk factors better than FMD. ${ }^{18}$ With respect to the MetSyn, there are limited and conflicting reports exploring the relationship of MetSyn and macrovascular and microvascular function, as assessed by brachial artery FMD, and leg or forearm blood flow. ${ }^{19-23}$ These studies suggest a possible association of the MetSyn with impaired resistance vessel function, ${ }^{20-22}$ but conflicting results with respect to conduit vessel function. ${ }^{19,22,23}$ Accordingly, the present study was designed to explore whether there is an association between the MetSyn, as defined by NCEP, and macrovascular and microvascular function in a large cohort of middle-age males without diabetes or CVD from the Firefighters and Their Endothelium (FATE) study. ${ }^{24}$ We hypothesized that the MetSyn would be associated with reduced function of both conduit and resistance vessels, as assessed by brachial artery FMD and HSS, respectively.

\section{Methods}

\section{FATE study participants}

FATE is an ongoing Canadian, multicenter, prospective, longitudinal study of middle-aged, male firefighters designed to explore the relationship between peripheral endothelial function and the development of CVD. ${ }^{24}$ Between March 1999 and October 2003, 1,578 firefighters (active and retired) were recruited from four Canadian centers (Calgary, Montreal, Hamilton, and Halifax). Participants from Calgary included firefighters of all ages, while the other cities included those older than 45 years. All individuals were screened to exclude those with a history of prior CVD, as previously described. ${ }^{24}$ Based on a survey of 300 FATE participants, they were relatively active with the average individual performing $14 \pm 2.3$ hours of light activity, $2.09 \pm 2.01$ hours of moderate activity, and $1.0 \pm 1.0$ hours of hard or very hard work during the week prior to enrollment into the study. The protocol was approved by the individual research ethics committees at all four sites and all participants gave written informed consent prior to the baseline assessment.

\section{Baseline assessment and classification of metabolic syndrome}

The baseline assessment was performed after a 12-hour overnight fast, and vasoactive medications (angiotensin-converting enzyme inhibitors, angiotensin receptor blockers, calcium channel blockers, beta-blockers, and nitrates) and alcohol were withheld for 48 hours prior to the visit. Smokers were asked to refrain from smoking for at least 4 hours before the visit and they were not studied within 5 days of smoke or fire exposure. Fasting blood samples were obtained at this visit for the baseline biochemical assessment. A physical exam, including blood pressure, height, weight, and waist circumference, was performed according to standardized methods and non-invasive vascular function was carried out at that time.

Of the 1,578 FATE participants, 1,480 had sufficient data to classify for the MetSyn according to the modified NCEP criteria ${ }^{3}$ and all had technically adequate vascular function tests (FMD and HSS). Of these, 63 patients with established diabetes mellitus (based on self-reported history, use of hypoglycemic agents, and/or a fasting glucose $>7 \mathrm{mmol} / \mathrm{l}$ at baseline) were excluded, leaving 1,417 individuals, which comprised the current study cohort. Participants were classified as having the MetSyn if they had three or more of the following criteria: abdominal obesity defined as waist circumference $>102 \mathrm{~cm}$; hypertriglyceridemia defined as triglycerides $>1.7 \mathrm{mmol} / \mathrm{l}$; low HDL cholesterol with an HDL $<1 \mathrm{mmol} / \mathrm{l}$; hypertension defined as systolic blood pressure $>130 \mathrm{mmHg}$ or diastolic blood pressure $>85 \mathrm{mmHg}$; and impaired fasting glucose defined by fasting glucose $>5.6 \mathrm{mmol} / \mathrm{l}$.

\section{Non-invasive assessment of vascular function}

Vascular function of the brachial artery was assessed non-invasively using a high-resolution vascular ultrasound system equipped with a $7.5-10-\mathrm{MHz}$ linear-array vascular transducer, according to well-described methods. ${ }^{14,15,24,25}$ Briefly, the right brachial artery was imaged longitudinally $2-15 \mathrm{~cm}$ above the antecubital crease. A pneumatic tourniquet, placed on the upper arm, was inflated to $250 \mathrm{mmHg}$ pressure for 5 minutes and rapidly deflated, resulting in reactive hyperemia. Brachial artery images were obtained before cuff inflation (first baseline), after cuff deflation (postreactive hyperemia), after 10 minutes of rest (second baseline), and 3 minutes after sublingual nitroglycerin $(0.3 \mathrm{mg})$ and were recorded on sVHS tapes. Additionally, pulsed-wave Doppler measurements were obtained before and immediately after cuff deflation to determine HSS.

Off-line analyses were performed at the Vascular Function Core Laboratory by a single, experienced 
technician, with extensive experience in FMD assessments, as previously described. ${ }^{25,26}$ Computerassisted edge detection brachial artery analysis software (DEA, Vasometrix, Montreal, Canada) was used to calculate brachial artery diameters, as previously described. ${ }^{25,26}$ FMD was defined as the maximal percent change in brachial artery diameter (between $60 \mathrm{~s}$ and $90 \mathrm{~s}$ ) after reactive hyperemia, compared to the first baseline. Nitroglycerinmediated dilatation (NMD) was calculated as the percent change in brachial artery diameter at $3 \mathrm{~min}$ utes after nitroglycerin compared to the second baseline.

Reactive hyperemic flow was measured using the recorded pulsed-wave Doppler measurements. The velocity time integral (VTI) in reactive hyperemia was determined by measuring two consecutive tracings of the velocity envelope obtained at peak flow immediately following cuff release and averaging them. One cardiac cycle was determined based on ECG gating. HSS in reactive hyperemia was calculated using the following equation: HSS $\left(\right.$ dyne $\left./ \mathrm{cm}^{2}\right)$ $=8 * 0.035\left(\right.$ dyne $\left.* \mathrm{~s} / \mathrm{cm}^{2}\right) *$ VTI $(\mathrm{cm}) *$ [heart ratel $60] /$ [baseline brachial artery diameter $(\mathrm{mm}) / 10$ ] We made an assumption of laminar flow and constant viscosity in our calculations, which is similar to the methods employed by others. ${ }^{18}$

The intraobserver and interobserver variability for repeat brachial artery diameter measurements at the Core Laboratory are $0 \pm 0.07 \mathrm{~mm}$ and $0.05 \pm$ $0.16 \mathrm{~mm}$, respectively. Among 51 participants undergoing repeat FMD testing, group means were similar $(8.2 \pm 3.2$ versus $8.3 \pm 2.8 \%)$, and the mean of the absolute difference between determinations for each participant was a favorable $1.8 \pm$ $1.6 \%$. For repeat HSS determinations, the coefficient of variation was $23 \%$.

\section{Biochemical analyses}

Plasma glucose and serum lipids were measured by standard automated analyzers. Plasma insulin levels were measured with a commercial radioimmunoassay kit (Pharmacia \& Upjohn Inc., Mississauga, ON, Canada); high-sensitivity C-reactive protein (hsCRP) levels were measured by a particleenhanced immunoturbidimetric method with a Roche/Hitachi 912 analyzer (Roche Diagnostics, Laval, Quebec, Canada). Insulin resistance was estimated using the homeostatic model assessmentinsulin resistance (HOMA-IR), which equaled: [plasma glucose $(\mathrm{mmol} / \mathrm{l}) *$ plasma insulin $(\mathrm{pmol} / \mathrm{l}) /$ $7.175] / 22.5 .{ }^{27}$

\section{Statistical analysis}

Continuous data are reported as means \pm standard deviation and categorical data are reported as counts and percentage, unless stated otherwise.
The dichotomous relationship between the presence or absence of MetSyn and HOMA-IR and hs-CRP was determined using the Wilcoxon rank-sum test. The dichotomous relationship between MetSyn and FMD, NMD, and HSS was determined using oneway analysis of variance. Adjustments for age, smoking status, and brachial artery baseline diameter were performed using multiple logistic regression analysis. The ordinal relationship between the number of NCEP MetSyn criteria and the mean values of FMD and HSS were determined using one-way analysis of variance. Simple linear regression was used to determine the relationship of insulin resistance (HOMA-IR) to FMD and HSS. Multiple regression analysis was used to determine whether specific NCEP MetSyn criteria, the presence of MetSyn (according to the NCEP definition), and insulin resistance (HOMA-IR) were independent predictors for FMD and HSS. Two-sided $p$-values $\leq 0.05$ were considered to indicate statistical significance.

\section{Results}

\section{Characteristics of the FATE participants and prevalence of metabolic syndrome}

The baseline clinical and biochemical characteristics of the FATE participants are summarized in Table 1, according to MetSyn status. Of the 1,417 male participants, $267(19 \%)$ met the NCEP definition for MetSyn. Those with MetSyn were older and had higher BMI, waist circumference, blood pressure, plasma glucose, insulin, HOMA-IR and hs-CRP levels and had proportionally more dyslipidemia, statins, angiotensin-converting enzyme inhibitors, and angiotensin receptor blockers, but fewer nonsmokers. Brachial artery diameters were significantly larger in the individuals with MetSyn.

\section{Relationship between metabolic syndrome and vascular function}

The relationship between MetSyn and brachial artery vasodilatory function, including brachial artery FMD and HSS, and NMD is summarized in Table 2. FMD was significantly lower in those with MetSyn $(p=0.02)$. However, those with MetSyn had many significant differences in baseline characteristics, many of which may be important confounders. While the differences in weight, BMI, waist circumference, blood pressure, lipids, glucose, insulin, HOMA-IR, and use of antihypertensive medications are expected in those with MetSyn, the differences in age, smoking, and brachial artery diameter may be confounding variables for FMD and HSS. Therefore, after adjusting for age, smoking and brachial artery diameter, we found that 
Table 1 Clinical and biochemical characteristics according to NCEP MetSyn status

\begin{tabular}{llc}
\hline & No MetSyn, $n=1150$ (81\%) & MetSyn, $n=267(19 \%)$ \\
\hline Age & $48.3 \pm 9.9$ & $52.5 \pm 8.3^{*}$ \\
Weight, kg & $87.6 \pm 10.0$ & $101.4 \pm 13.0^{*}$ \\
BMI, kg/m² & $27.6 \pm 3.0$ & $31.7 \pm 3.6^{*}$ \\
Waist circumference, cm & $94.3 \pm 8.0$ & $106.3 \pm 8.7^{*}$ \\
Systolic blood pressure, mmHg & $125 \pm 15.0$ & $138 \pm 17.0^{*}$ \\
Diastolic blood pressure, $\mathrm{mmHg}$ & $80 \pm 9.0$ & $88 \pm 10.0^{*}$ \\
Total cholesterol, mmol/l & $5.2 \pm 1.0$ & $5.5 \pm 1.0^{*}$ \\
LDL cholesterol, mmol/l & $3.3 \pm 0.8$ & $3.3 \pm 0.9$ \\
HDL cholesterol, mmol/l & $1.3 \pm 0.3$ & $1.1 \pm 0.2^{*}$ \\
Brachial artery diameter, mm & $4.3 \pm 0.5$ & $4.4 \pm 0.6^{*}$ \\
Triglycerides, mmol/l & $1.2(0.87-1.63)$ & $2.3(1.83-2.96)^{*}$ \\
Fasting glucose, mmol/l & $5.1(4.8-5.4)$ & $5.6(5.1-5.9)^{*}$ \\
Insulin, pmol/l & $44.4(32.7-60.9)$ & $84.3(62.9-115.4)^{*}$ \\
HOMA-IR & $1.39(1.01-2.01)$ & $2.88(2.05-4.05)^{*}$ \\
hs-CRP, mg/l & $1.1(0.6-2.0)$ & $2.1(1.1-3.3)^{*}$ \\
Smoking: current/ex/non & $141 / 449 / 560(12 / 39 / 49)$ & $28 / 140 / 99^{*}(11 / 52 / 37)$ \\
Statins & $70(6)$ & $27(10)^{*}$ \\
ACE/ARB & $50(4)$ & $30(11)^{*}$ \\
\hline
\end{tabular}

Data given as mean $\pm \mathrm{SD}$, median $(25-75 \%$ range) and $n(\%)$.

${ }^{*} p<0.05$

MetSyn, metabolic syndrome; SD, standard deviation; BMI, body mass index; LDL, low-density lipoprotein; HDL, highdensity lipoprotein; HOMA-IR, homeostatic model assessment-insulin resistance; hs-CRP, high-sensitivity C-reactive protein; $A C E$, angiotensin-converting enzyme inhibitor; $A R B$, angiotensin receptor blocker.

FMD was not significantly different in those with MetSyn (adjusted $p$-value $=0.2$ ). Alternatively, HSS was significantly lower in individuals with MetSyn, both unadjusted and adjusted for baseline differences in age, smoking and brachial artery diameters ( $p<0.001$ and $p=0.01$, respectively). NMD was similar in those with and without MetSyn (nonadjusted $p=0.1$; adjusted $p=0.3$ ).

There was no relationship between the number of NCEP MetSyn criteria and FMD (mean FMD for $0,1,2,3,4$, and 5 criteria: $8.7 \pm 3.9,8.7 \pm 4.1,8.7 \pm$ $4.0,8.0 \pm 3.8,8.1 \pm 4.9,8.3 \pm 3.4 \% ; p=0.4$ for trend). In contrast, there was a significant, inverse graded relationship with the number of NCEP criteria and mean HSS (mean HSS for 0, 1, 2, 3, 4, and 5 criteria: $83.2 \pm 22.5,82.2 \pm 24.7,76.5 \pm 27.2$, $74.3 \pm 27.4,66.5 \pm 28.4,67.1 \pm 27.6 \mathrm{dyne} / \mathrm{cm}^{2}$ $p<0.001$ for trend).

\section{Relationships of FMD and HSS and metabolic syndrome risk factors}

Multiple regression analysis between FMD as the dependent variable and the five individual NCEP MetSyn criteria as independent dichotomous variables is shown in Table 3 (Model 1a). The presence or absence of the NCEP-defined MetSyn was added to the model to determine whether the diagnosis of MetSyn provided any predictive value beyond the individual MetSyn criteria (Model 2a). As shown in the table, none of the individual MetSyn criteria, nor the overall presence of MetSyn, was significant independent predictors for FMD. Multiple regression analysis between HSS as the dependent variable and the five individual NCEP MetSyn criteria as independent dichotomous variables is shown in Table 3 (Model 1b). Of the individual NCEP criteria, abdominal obesity, systolic hypertension, and

Table 2 Relationship of brachial artery endothelium-dependent FMD, HSS, and endothelium-independent NMD and NCEP MetSyn status

\begin{tabular}{lcccc}
\hline & $\begin{array}{l}\text { No MetSyn, } n=1150 \\
(81 \%)\end{array}$ & $\begin{array}{l}\text { MetSyn, } n=267 \\
(19 \%)\end{array}$ & $p$-value & Adjusted $p$-value* \\
\hline \%FMD & $8.7 \pm 4.0$ & $8.1 \pm 4.1$ & 0.02 & 0.2 \\
\%NMD & $15.8 \pm 5.8$ & $15.2 \pm 6.0$ & 0.1 & 0.3 \\
HSS (dyne/cm $\left.{ }^{2}\right)$ & $80.9 \pm 24.8$ & $72.0 \pm 27.8$ & $<0.001$ & 0.01 \\
\hline
\end{tabular}

*Adjusted for age, smoking status and baseline brachial artery diameter.

MetSyn, metabolic syndrome; FMD, flow-mediated dilatation; NMD, nitroglycerin-mediated dilatation; HSS, hyperemic shear stress. 
Table 3 Multiple regression models relating macrovascular endothelial function (FMD) or microvascular endothelial function (HSS) as the dependent variable and (1) the five individual NCEP MetSyn criteria as independent variables (Model 1) and (2) the five individual NCEP MetSyn criteria along with the presence of MetSyn (Model 2)

\begin{tabular}{|c|c|c|c|c|c|c|c|c|}
\hline \multirow[t]{3}{*}{ Independent variable (categorical) } & \multicolumn{4}{|c|}{$\begin{array}{l}\text { Macrovascular endothelial function } \\
\text { FMD }\end{array}$} & \multicolumn{4}{|c|}{$\begin{array}{c}\text { Microvascular endothelial function } \\
\text { HSS }\end{array}$} \\
\hline & \multicolumn{2}{|c|}{$\begin{array}{l}\text { Model } 1 \mathrm{a} \\
\mathrm{R}^{2}=0.005 \\
\quad p=0.3\end{array}$} & \multicolumn{2}{|c|}{$\begin{array}{c}\text { Model } 2 \mathrm{a} \\
\mathrm{R}^{2}=0.007 \\
p=0.2\end{array}$} & \multicolumn{2}{|c|}{$\begin{array}{l}\text { Model 1b; } \\
\mathrm{R}^{2}=0.05 \\
p<0.00001\end{array}$} & \multicolumn{2}{|c|}{$\begin{array}{l}\text { Model 2b, } \\
\mathrm{R}^{2}=0.05 \\
p<0.00001\end{array}$} \\
\hline & $\begin{array}{l}\text { Beta } \\
\text { coeffi- } \\
\text { cient }\end{array}$ & $p$-value & $\begin{array}{l}\text { Beta } \\
\text { coeffi- } \\
\text { cient }\end{array}$ & $p$-value & $\begin{array}{l}\text { Beta } \\
\text { coeffi- } \\
\text { cient }\end{array}$ & $p$-value & $\begin{array}{l}\text { Beta } \\
\text { coeffi- } \\
\text { cient }\end{array}$ & $p$-value \\
\hline Metabolic syndrome (present or absent) & - & - & -0.075 & 0.08 & - & - & -0.017 & 0.7 \\
\hline Abdominal obesity (waist $>102 \mathrm{~cm}$ ) & 0.006 & 0.8 & 0.036 & 0.3 & -0.114 & $<0.001$ & -0.107 & 0.001 \\
\hline $\begin{array}{l}\text { Hypertriglyceridemia } \\
\text { (triglycerides }>1.7 \mathrm{mmol} / \mathrm{l} \text { ) }\end{array}$ & 0.007 & 0.8 & 0.028 & 0.4 & 0.013 & 0.7 & 0.018 & 0.6 \\
\hline $\begin{array}{l}\text { Low high-density lipoprotein cholesterol } \\
\qquad(\mathrm{HDL}<1 \mathrm{mmol} / \mathrm{l})\end{array}$ & -0.019 & 0.5 & 0.005 & 0.9 & -0.017 & 0.5 & -0.013 & 0.7 \\
\hline Hypertension (>130 mmHg systolic) & 0.006 & 0.8 & 0.011 & 0.7 & -0.109 & $<0.001$ & -0.108 & $<0.001$ \\
\hline Hypertension (>85 mmHg diastolic) & -0.053 & 0.08 & -0.042 & 0.2 & -0.033 & 0.3 & -0.031 & 0.3 \\
\hline $\begin{array}{l}\text { Impaired fasting glucose } \\
\quad \text { (glucose }>5.6 \mathrm{mmol} / \mathrm{l})\end{array}$ & -0.041 & 0.1 & -0.020 & 0.5 & -0.070 & 0.008 & -0.066 & 0.02 \\
\hline
\end{tabular}

FMD, flow-mediated dilatation; HSS, hyperemic shear stress.

impaired fasting glucose were significantly and independently associated with HSS. The presence or absence of the NCEP-defined MetSyn was added as an additional independent variable in the model (Model 2b) and we found that the overall presence of the MetSyn was not independently associated with HSS beyond the individual components of the syndrome $(p=0.7)$.

\section{Relationships of FMD and HSS and insulin resistance}

Using linear regression, we found no significant relationship between insulin resistance (HOMA-IR as a continuous variable) and FMD $(r=-0.026$; $p=0.3$ ), while there was a significant inverse rela- tionship with HSS $(r=-0.15 ; p<0.01)$. Multiple regression analysis between FMD (vs HSS) as the dependent variable and the five individual MetSyn criteria plus insulin resistance (HOMA-IR > upper quartile [2.38]) as dichotomous independent variables are shown in Table 4. None of the individual MetSyn criteria, nor the presence of insulin resistance were significant independent predictors for FMD. Conversely, abdominal obesity, systolic hypertension, and impaired fasting glucose were significantly and independently associated with HSS, while insulin resistance was not $(p=0.06)$. Similar findings were found when insulin resistance was included in the model as a continuous variable (not shown).

Table 4 Multiple regression models relating macrovascular endothelial function (FMD) or microvascular endothelial function (HSS) as the dependent variable and the five individual NCEP MetSyn criteria and insulin resistance (HOMA$\mathrm{IR}>$ upper quartile) as independent variables

\begin{tabular}{|c|c|c|c|c|}
\hline \multirow[t]{2}{*}{ Independent variable (categorical) } & \multicolumn{2}{|c|}{$\begin{array}{l}\text { Macrovascular endothelial } \\
\text { function } \\
\text { FMD } \\
\mathrm{R}^{2}=0.005 ; p=0.4\end{array}$} & \multicolumn{2}{|c|}{$\begin{array}{c}\text { Microvascular endothelial } \\
\text { function } \\
\text { HSS } \\
\mathrm{R}^{2}=0.05 ; p<0.00001\end{array}$} \\
\hline & $\begin{array}{l}\text { Beta } \\
\text { coefficient }\end{array}$ & $p$-value & $\begin{array}{l}\text { Beta } \\
\text { coefficient }\end{array}$ & $p$-value \\
\hline Abdominal obesity (waist > $102 \mathrm{~cm}$ ) & 0.012 & 0.7 & -0.094 & 0.001 \\
\hline Hypertriglyceridemia (triglycerides $>1.7 \mathrm{mmol} / \mathrm{l}$ ) & 0.010 & 0.7 & 0.023 & 0.4 \\
\hline Low high-density lipoprotein cholesterol (HDL $<1 \mathrm{mmol} / \mathrm{l})$ & -0.018 & 0.5 & -0.013 & 0.6 \\
\hline Hypertension (>130 mmHg systolic) & 0.007 & 0.8 & -0.107 & 0.0001 \\
\hline Hypertension (>85 mmHg diastolic) & -0.053 & 0.09 & -0.032 & 0.3 \\
\hline Impaired fasting glucose (glucose $>5.6 \mathrm{mmol} / \mathrm{l}$ ) & -0.036 & 0.2 & -0.055 & 0.048 \\
\hline Insulin resistance (HOMA-IR > upper quartile [2.38]) & -0.019 & 0.5 & 0.060 & 0.06 \\
\hline
\end{tabular}

FMD, flow-mediated dilatation; HSS, hyperemic shear stress. 


\section{Discussion}

In this large cohort of middle-aged male firefighters without established diabetes or cardiovascular disease, the prevalence of NCEP-defined MetSyn was $19 \%$. After adjusting for relevant covariates, we found no association between MetSyn and conduit function, as assessed by FMD. On the other hand, HSS, a measure of resistance vessel function, was significantly lower in the presence of MetSyn, with an inverse, graded relationship to the number of NCEP criteria present. While the individual NCEP criteria of abdominal obesity, systolic hypertension, and impaired fasting glucose were independently associated with HSS, the presence of the MetSyn itself provided no additional predictive value beyond these individual components. Moreover, this association did not appear to be significantly related to insulin resistance, as assessed by HOMA-IR.

The lack of an independent association between brachial artery FMD and MetSyn, after adjusting for confounding variables, is consistent with a small study from Wendelhag, et al. ${ }^{19}$ and with the larger study by Lind, ${ }^{22}$ which used similar methodology to our study. That is, Wendelhag, et al. ${ }^{19}$ found that FMD was similar in those with and without MetSyn (defined by the World Health Organization) among males over the age of $60(n=55)$. Similarly, Lind ${ }^{22}$ found no relationship between FMD and the presence of the NCEP-defined MetSyn among 1,016 individuals aged 70 years (median FMD: $4.1 \%$ vs $4.5 \% ; p=0.6$ ), which agree with our negative findings in a younger population of men. In contrast, the recent Framingham Offspring study involving 2,123 middle-aged to elderly individuals demonstrated a significant inverse relationship between the presence of MetSyn and FMD. ${ }^{23}$ However, similar to our study, they demonstrated larger baseline brachial artery diameters with MetSyn and although the baseline diameter is inversely related to FMD they did not adjust for this covariable. Therefore, one cannot be sure whether this confounding variable was partly driving the positive association between FMD and MetSyn. Thus, based on our study and the other large studies, ${ }^{22,23}$ it remains controversial as to whether there is an association between FMD and MetSyn.

Unlike FMD, we found that MetSyn was associated with lower HSS, and that there was an inverse, graded relationship with the number of NCEP criteria present. While it has been generally accepted that FMD is dependent in part upon the endothelial release of nitric oxide (NO), HSS, the stimulus for FMD, has often been regarded as endotheliumindependent. ${ }^{17,28}$ That is, the extent of reactive hyperemia in response to forearm ischemia, has been long considered to be related to the local release of ischemia-induced vasodilators. ${ }^{17,28}$ However, some studies have suggested that hyperemic flow may in part be dependent upon the endothelial release of $\mathrm{NO}$, as the NO synthase inhibitor $N^{\mathrm{G}_{-}}$ monomethyl-L-arginine has been shown to partially reduce the extent of hyperemic flow after transient ischemia. ${ }^{29,30}$ However, this has not been confirmed by other ultrasound studies. ${ }^{31}$ Recently, Mitchell, et al. ${ }^{18}$ measured brachial artery FMD and diastolic shear stress produced during reactive hyperemia and reported that coronary risk factors were better related to HSS than to FMD. They hypothesized that the impaired FMD responses attributed to coronary risk factors may be partly related to a reduced stimulus for dilatation, reflecting microvascular dysfunction, rather than impaired conduit artery function. ${ }^{18}$ Thus, our findings suggest that the MetSyn is associated with impaired microvascular function, which is supported by previous studies that have directly measured microvascular function. ${ }^{20-22}$ Lteif, et al. $^{20}$ demonstrated that there was impaired microvascular function in 97 subjects with MetSyn. Similarly, Lind ${ }^{22}$ found that forearm blood flow responses to acetylcholine were impaired in individuals with MetSyn and that this was inversely related to the number of NCEP criteria present. Hamburg, et $a .^{23}$ similarly demonstrated that MetSyn was inversely related to brachial artery microvascular function, as assessed by Doppler-based measurements of hyperemic flow velocity. In agreement with the studies of Lteif, et al. ${ }^{20}$ and Lind, ${ }^{22}$ we found that this impairment in microvascular function was specifically related to abdominal obesity, hypertension, and dysglycemia. Unlike Lteif, et al. ${ }^{20}$ and Hamburg, et al. ${ }^{23}$ we found no independent association of insulin resistance with HSS, beyond the NCEP components. These individual components of the metabolic syndrome have all been previously reported to be associated with endothelial dysfunction. ${ }^{23}$ Discussion of the pathophysiologic mechanisms whereby these factors may contribute to vascular dysfunction is beyond the scope of this paper, but have been well discussed previously. ${ }^{12,13}$ The lower HSS associated with MetSyn may have clinical significance, as this measure of microvascular function has been associated with coronary risk factors $^{18}$ and has recently been shown to be predictive of future cardiovascular events in patients with peripheral vascular disease undergoing vascular surgery. ${ }^{32}$

That we saw an association between MetSyn and microvascular function but no relationship with macrovascular function is consistent with the human study of Lind ${ }^{22}$ in which macro- and microvascular function was separately studied by noninvasive and invasive methods. This may suggest that the MetSyn may have differential effects on conduit and resistance vessels, with vascular dysfunction appearing primarily in the resistance 
vessels, which has been previously described in an animal model of insulin resistance. ${ }^{33}$ O'Brien, et al., ${ }^{33}$ using a JCR:LA-cp rat model of insulin resistance, found that resistance vessels of these insulin-resistant rats had vascular dysfunction with impaired response to acetylcholine, while conduit vessels maintained normal responses to acetylcholine. However, as other animal models of insulin resistance have demonstrated impaired conduit vessel function, these findings may be modeldependent. ${ }^{34}$ Moreover, demonstrating defects in macrovascular function may be dependent upon the type of human individuals that are assessed. For example, Beckman, et al. ${ }^{35}$ have shown that FMD varies according to the type of insulin resistance that is present, as only individuals with type 2 diabetes had impaired FMD, while those with nonHIV lipodystrophic diabetes and polycystic ovary syndrome did not have impaired conduit vessel function despite having profound insulin resistance. Multivariate analysis in our study suggests the impairment of microvascular function is a function of the abdominal obesity, hypertension, and dysglycemia that are components of the MetSyn and were found in our participants. Similar to the recent study by Hamburg, et al. ${ }^{23}$ we found that the association between MetSyn and microvascular function was predominately driven by the presence of the individual components, rather than the overall clustering of risk factors that define MetSyn, which has been also noted in some epidemiologic studies. ${ }^{4}$

Our study has certain limitations. Most importantly, our population is confined to males who are relatively healthy, free of overt CVD and diabetes, and have normal FMDs. Potentially, this may bias our study against demonstrating a relationship of FMD to various risk factors. Second, one may question the accuracy of the FMD measurements from a multicenter study. However, all the FMD studies were acquired at centers with extensive experience in performing FMD and the analysis was performed by an experienced core laboratory, and we noted good reproducibility on repeat studies. Lastly, although we found significant associations with various components of the MetSyn and HSS, the contribution appears to be relatively small in our model. These limitations are offset by the strength of the study, namely the large sample size which provides sufficient power to look at the relationship between MetSyn and vascular function, while correcting for baseline differences in the population.

In summary, we demonstrated that the MetSyn is associated with lower HSS, which may represent impaired microvascular vasodilator function. This reduction in shear stress was better related to the individual components of MetSyn, including abdominal obesity, systolic hypertension, and dysglycemia, rather than to the overall syndrome per se.
Thus, these specific MetSyn components may contribute to the development of CVD by causing microvascular vasodilator dysfunction.

\section{Acknowledgements}

This study was supported by grants from the Heart and Stroke Foundation of Alberta, Canadian Institute of Health Research, and Pfizer Canada.

\section{Disclosure}

There are no conflicts of interest.

\section{References}

1 Reaven, GM. Banting lecture 1988. Role of insulin resistance in human disease. Diabetes 1988; 37: 1595-1607.

2 Third Report of the National Cholesterol Education Program (NCEP) Expert Panel on Detection. Evaluation, and Treatment of High Blood Cholesterol in Adults (Adult Treatment Panel III) final report. Circulation 2002; 106: 3143-3421.

3 Grundy, SM, Brewer, HB, Cleeman, JI, Smith, SC, Lenfant, C. Definition of metabolic syndrome: report of the National Heart, Lung, and Blood Institute/American Heart Association conference on scientific issues related to definition. Circulation 2004; 109: 433-438.

4 Kahn, R, Buse, J, Ferrannini, E, Stern, M. The metabolic syndrome: time for a critical appraisal. Joint statement from the American Diabetes Association and the European Association for the Study of Diabetes. Diabetologia 2005; 48: 1684-1699.

5 Grundy, SM. Metabolic syndrome: connecting and reconciling cardiovascular and diabetes worlds. $\mathrm{J}$ Am Coll Cardiol 2006; 47: 1093-1100.

6 Lakka, HM, Laaksonen, DE, Lakka, TA, et al. The metabolic syndrome and total and cardiovascular disease mortality in middle-aged men. JAMA 2002; 288: 2709-2716.

7 Lorenzo, C, Okoloise, M, Williams, K, Stern, MP, Haffner, SM. The metabolic syndrome as predictor of type 2 diabetes: the San Antonio heart study. Diabetes Care 2003; 26: 3153-3159.

8 Sattar, N, Gaw, A, Scherbakova, O, et al. Metabolic syndrome with and without $\mathrm{C}$-reactive protein as a predictor of coronary heart disease and diabetes in the West of Scotland Coronary Prevention Study. Circulation 2003; 108: 414 419.

9 Tzou, WS, Douglas, PS, Srinivasan, SR, et al. Increased subclinical atherosclerosis in young adults with metabolic syndrome: the Bogalusa Heart Study. J Am Coll Cardiol 2005; 46: 457-463.

10 Reilly, MP, Wolfe, ML, Rhodes, T, Girman, C, Mehta, N, Rader, DJ. Measures of insulin resistance add incremental value to the clinical diagnosis of metabolic syndrome in association with coronary atherosclerosis. Circulation 2004; 110: 803-809. 
11 Solymoss, BC, Bourassa, MG, Lesperance, J, et al. Incidence and clinical characteristics of the metabolic syndrome in patients with coronary artery disease. Coron Artery Dis 2003; 14: 207-212.

12 Lteif, A, Mather, K. Insulin resistance, metabolic syndrome and vascular diseases: update on mechanistic linkages. Can J Cardiol 2004; 20(suppl B): 66B-76B.

13 Kim, JA, Montagnani, M, Koh, KK, Quon, MJ. Reciprocal relationships between insulin resistance and endothelial dysfunction: molecular and pathophysiological mechanisms. Circulation 2006; 113: 1888-1904.

14 Celermajer, DS, Sorensen, KE, Gooch, VM, et al. Noninvasive detection of endothelial dysfunction in children and adults at risk of atherosclerosis. Lancet 1992; 340: $1111-1115$.

15 Corretti, MC, Anderson, TJ, Benjamin, EJ, et al. Guidelines for the ultrasound assessment of endothelialdependent flow-mediated vasodilation of the brachial artery: a report of the International Brachial Artery Reactivity Task Force. J Am Coll Cardiol 2002; 39: 257-265.

16 Yeboah, J, Crouse, JR, Hsu, FC, Burke, GL, Herrington, DM. Brachial flow-mediated dilation predicts incident cardiovascular events in older adults: the Cardiovascular Health Study. Circulation 2007; 115: 2390-2397.

17 Joannides, R, Bellien, J, Thuillez, C. Clinical methods for the evaluation of endothelial function - a focus on resistance arteries. Fundam Clin Pharmacol 2006; 20: 311-320.

18 Mitchell, GF, Parise, H, Vita, JA, et al. Local shear stress and brachial artery flow-mediated dilation: the Framingham Heart Study. Hypertension 2004; 44: 134-139.

19 Wendelhag, I, Fagerberg, B, Hulthe, J, Bokemark, L, Wikstrand, J. Endothelium-dependent flow-mediated vasodilatation, insulin resistance and the metabolic syndrome in 60-year-old men. J Intern Med 2002; 252: 305-313.

20 Lteif, AA, Han, K, Mather, KJ. Obesity, insulin resistance, and the metabolic syndrome: determinants of endothelial dysfunction in whites and blacks. Circulation 2005; 112: 32-38.

21 Bahia, L, Aguiar, LG, Villela, N, et al. Relationship between adipokines, inflammation, and vascular reactivity in lean controls and obese subjects with metabolic syndrome. Clinics 2006; 61: 433-440.

22 Lind, L. Endothelium-dependent vasodilation, insulin resistance and the metabolic syndrome in an elderly cohort The Prospective Investigation of the Vasculature in Uppsala Seniors (PIVUS) study. Atherosclerosis 2008; 196: 795-802.

23 Hamburg, NM, Larson, MG, Vita, JA, et al. Metabolic syndrome, insulin resistance, and brachial artery vasodilator function in Framingham Offspring participants without clinical evidence of cardiovascular disease. Am J Cardiol 2008; 101: 82-88.
24 Anderson, TJ, Roberts, AC, Hildebrand, K, et al. The fate of endothelial function testing: rationale and design of the Firefighters And Their Endothelium (FATE) study. Can J Cardiol 2003; 19: 61-66.

25 Yan, RT, Anderson, TJ, Charbonneau, F, Title, L, Verma, $\mathrm{S}$, Lonn, E. Relationship between carotid artery intimamedia thickness and brachial artery flow-mediated dilation in middle-aged healthy men. J Am Coll Cardiol 2005; 45: 1980-1986.

26 Anderson, TJ, Elstein, E, Haber, H, Charbonneau, F. Comparative study of ACE-inhibition, angiotensin II antagonism, and calcium channel blockade on flowmediated vasodilation in patients with coronary disease (BANFF study). J Am Coll Cardiol 2000; 35: 60-66.

27 Bonora, E, Targher, G, Alberiche, M, et al. Homeostasis model assessment closely mirrors the glucose clamp technique in the assessment of insulin sensitivity: studies in subjects with various degrees of glucose tolerance and insulin sensitivity. Diabetes Care 2000; 23: 57-63.

28 Loscalzo, J, Vita, JA. Ischemia, hyperemia, exercise, and nitric oxide. Complex physiology and complex molecular adaptations. Circulation 1994; 90: 2556-2559.

29 Meredith, IT, Currie, KE, Anderson, TJ, Roddy, MA, Ganz, P, Creager, MA. Postischemic vasodilation in human forearm is dependent on endothelium-derived nitric oxide. Am J Physiol 1996; 270: H1435-H1440.

30 Higashi, Y, Sasaki, S, Nakagawa, K, Matsuura, H, Kajiyama, G, Oshima, T. A noninvasive measurement of reactive hyperemia that can be used to assess resistance artery endothelial function in humans. Am J Cardiol 2001; 87: 121-125, A9.

31 Joannides, R, Haefeli, WE, Linder, L, et al. Nitric oxide is responsible for flow-dependent dilatation of human peripheral conduit arteries in vivo. Circulation 1995; 91: 1314 1319.

32 Huang, AL, Silver, AE, Shvenke, E, et al. Predictive value of reactive hyperemia for cardiovascular events in patients with peripheral arterial disease undergoing vascular surgery. Arterioscler Thromb Vasc Biol 2007; 27: 2650-2656.

33 O'Brien, SF, McKendrick, JD, Radomski, MW, Davidge, ST, Russell, JC. Vascular wall reactivity in conductance and resistance arteries: differential effects of insulin resistance. Can J Physiol Pharmacol 1998; 76: 72-76.

34 McNamee, CJ, Kappagoda, CT, Kunjara, R, Russell, JC. Defective endothelium-dependent relaxation in the JCR:LA-corpulent rat. Circ Res 1994; 74: 1126-1132.

35 Beckman, JA, Goldfine, AB, Dunaif, A, Gerhard-Herman, M, Creager, MA. Endothelial function varies according to insulin resistance disease type. Diabetes Care 2007; 30: 1226-1232. 